\title{
Comorbidities as an Indication for Metabolic Surgery
}

\author{
Anne-Catherine Schwarz ${ }^{a} \quad$ Adrian T. Billeter $^{a} \quad$ Katharina M. Scheurlen $^{a} \quad$ Matthias Blüher $^{b}$ \\ Beat P. Müller-Stich ${ }^{a}$
}

${ }^{a}$ Department of General, Visceral, and Transplantation Surgery, University of Heidelberg, Heidelberg, Germany;

${ }^{b}$ Department of Medicine, Endocrinology and Nephrology, University of Leipzig, Leipzig, Germany

\section{Keywords}

Diabetes - Type 2 diabetes mellitus, T2DM .

Non-alcoholic steatohepatitis, NASH .

Edmonton Obesity Staging System, EOSS .

Bariatric surgery · Metabolic surgery · Sleeve ·

Roux-en-Y gastric bypass, RYGB · Gastric bypass

\section{Summary}

Metabolic diseases, comprising type 2 diabetes mellitus (T2DM), dyslipidemia, and non-alcoholic steatohepatitis (NASH), are rapidly increasing worldwide. Conservative medical therapy, including the newly available drugs, has only limited effects and does neither influence survival or the development of micro- or macrovascular complications, nor the progression of NASH to liver cirrhosis, nor the development of hepatocellular carcinomas in the NASH liver. In contrast, metabolic surgery is very effective independent of the preoperative body mass index (BMI) in reducing overall and cardiovascular mortality in patients with T2DM. Furthermore, metabolic surgery significantly reduces the development of microand macrovascular complications while being the most effective therapy in order to achieve remission of T2DM and to reach the targeted glycemic control. Importantly, even existing diabetic complications such as nephropathy as well as the features of NASH can be reversed by metabolic surgery. Here, we propose indications for metabolic surgery due to T2DM and NASH based on a simple but objective, disease-specific staging system. We outline the use of the Edmonton Obesity Staging System (EOSS) as a clinical staging system independent of the $\mathrm{BMI}$ that will identify patients who will benefit the most from metabolic surgery.

(C) 2018 S. Karger GmbH, Freiburg

\section{KARGER}

(c) 2018 S. Karger GmbH, Freiburg

\section{Introduction}

The prevalence of overweight and obesity is ever increasing, both in industrialized countries and developing nations. Despite its high frequency, the pathomechanisms causing obesity remain poorly understood; currently available conservative and medical therapies regularly fail and rarely achieve the desired long-term results. In the last 15 years, bariatric surgery has emerged as the most effective therapy for obesity, and it is the only therapy that consistently achieves long-term weight loss. While various bariatric surgical techniques have been developed over time, sleeve gastrectomy (SG) and Roux-en-Y gastric bypass (RYGB) are the most widely used today, since they combine a high level of safety with a good quality of life and long-lasting effects on obesity.

By the 1920s and 1930s, initial observations showed that type 2 diabetes mellitus (T2DM) improved after gastric surgery. In 1995, Pories et al. [1] also showed that bariatric surgery is an effective therapy for T2DM. Subsequent studies, in particular the longterm results of the Swedish Obese Subjects (SOS) study, further confirmed that bariatric surgery reduces the incidence of newonset T2DM while also effectively remitting existing T2DM [24]. Further randomized controlled trials (RCTs) comparing metabolic surgery with medical therapy showed that these surgical procedures were not only safe but also more effective than even intensive medical therapy in treating obesity and its comorbidities, and that they resulted in a better quality of life [3-6]. Beneficial effects of surgery on T2DM and other components of the metabolic syndrome, such as blood pressure and dyslipidemia, were also observed. Most importantly, metabolic surgery reduced overall as well as cardiovascular mortality. The latest studies indicate that metabolic surgery may even induce the cure of existing end-organ damage, e.g. diabetic microvascular complications such as nephropathy, neuropathy, and retinopathy, or other obesity-associated diseases such as non-alcoholic steatohepatitis (NASH) [7-9]. 


\begin{tabular}{|c|c|c|c|c|c|}
\multicolumn{7}{c}{ Obesity } \\
\cline { 2 - 6 } & \multicolumn{1}{c|}{\begin{tabular}{l} 
Stage 0 \\
\cline { 2 - 6 }
\end{tabular}} & Stage 1 & Stage 2 & Stage 3 & Stage 4 \\
\hline Medical & absent & $\begin{array}{c}\text { pre-clinical } \\
\text { risk factors }\end{array}$ & comorbidity & $\begin{array}{c}\text { end-organ } \\
\text { damage }\end{array}$ & end-stage \\
\hline Mental & absent & mild & moderate & severe & end-stage \\
\hline Functional & absent & mild & moderate & severe & end-stage \\
\hline
\end{tabular}

Fig. 1. Edmonton Obesity Staging System (EOSS) (modified from [16]).

Further studies in patients not fulfilling the BMI(body mass index)-based criteria for bariatric surgery, i.e. those with a BMI $<35$ $\mathrm{kg} / \mathrm{m}^{2}$, clearly show that metabolic procedures have similar beneficial effects on T2DM and other obesity-associated comorbidities in non-obese patients $[3,4]$. Therefore, the relevance of the currently accepted criteria for bariatric surgery, which were established by the National Institute of Health in 1992 and have not been revised since, is increasingly questioned. Studies from our institution and others over the last few years have demonstrated that metabolic surgery, or the use of bariatric procedures to treat metabolic diseases, is an effective therapy for metabolic disease regardless of a patient's body weight/BMI. Furthermore, research demonstrates that metabolic surgical procedures also improve existing diabetic complications such as neuropathy and nephropathy [7-10]. In contrast to these findings, concerning non-severely obese patients with severe metabolic disease, there is also ample evidence that patients with a high degree of obesity can be metabolically healthy [11-13]. These findings beg the question if comorbidities like T2DM, metabolic syndrome, NASH, and others would be more appropriate indications for metabolic surgery than a BMI $>40 \mathrm{~kg} / \mathrm{m}^{2}$. The new national German guidelines (S3-Leitlinien) for the 'Surgical Treatment of Obesity and Metabolic Diseases' address this overwhelming evidence and extend the indications for metabolic surgery, especially in patients with T2DM [14]. Noteworthy, the concept of such a benign obesity has been questioned by data that even 'metabolically healthy obese' individuals have an increased risk of cardiovascular disease compared to lean and healthy people [15].

Therefore, the purpose of this article is to outline possible indications for metabolic surgery independent of BMI, including T2DM, NASH, diabetic microvascular complications, and obesityrelated glomerulopathy.

\section{Assessment of Patients Suitable for Metabolic Surgery}

In 2009, Sharma and Kushner [16] introduced the newly-developed Edmonton Obesity Staging System (EOSS), as previously used scoring systems based solely on BMI or waist circumference were found to be insufficient for clinical practice. The EOSS is not based on the degree of obesity according to BMI but on the absence or presence - as well as the severity - of comorbidities and organ dysfunction. Figure 1 shows the five EOSS classification groups, which are based on the patients' medical, mental, and functional status.
A study from 2011 using data from the National Health and Human Nutrition Examination Surveys (NHANES) showed that the EOSS is a better predictor for mortality than BMI [17]. Survival curves using the EOSS diverged between stages $0-1$ and 2 and, in particular, compared to patients with EOSS stage 3 but did not differ when BMI grades were used. Padwal et al. [17] promulgate the benefit of this scoring system for clinical utility in assessing and stratifying each patient's obesity-associated risks.

The EOSS, however, has not been validated in a prospective clinical study. Furthermore, an important question is whether a patient with an EOSS stage 2 or even stage 3 can be reversed to a stage 1 or 0 by an intervention, be it conservative, medical, or surgical. Current studies regarding T2DM remission and the improvement of diabetic microvascular complications in particular suggest that the EOSS stage for each individual patient is, at least to some degree, reversible. Furthermore, the ideal time point for considering metabolic surgery needs to be defined and likely also depends on the comorbidities present. T2DM is a good example: patients with long-term T2DM and a need for insulin therapy have a much lesser likelihood for diabetes remission than patients with an impaired glucose tolerance $[2,18,19]$. Based on the EOSS, a patient with insulin therapy and a high insulin dose would be graded as EOSS 2 or 3, depending on the severity of the diabetic complications. In contrast, a patient with impaired glucose tolerance would be graded as EOSS 1. Hence, one could argue that even patients with T2DM and EOSS 1 should be considered for metabolic surgery since they would be likely to receive the strongest benefit. The importance of operating earlier rather than later is further supported by the findings of the SOS study, which shows that metabolic surgery has a lower impact on the reduction of macro- and microvascular events the longer the T2DM existed prior to surgery $[2,18]$. In contrast, a recent meta-analysis did not find a relationship between the duration of T2DM and the efficacy of metabolic operations regarding the reduced incidence or improvement of microvascular complications [8]. Lastly, a recent study by Ahlqvist et al. [20] found that there seem to be several subtypes of T2DM with different underlying causes such as high insulin resistance or insulin deficiency, and that these pose differing risks for the development of T2DM-related complications. Further studies are needed to confirm these findings, and the effects of metabolic surgery on these T2DM subtypes also need to be determined to personalize treatment and achieve the best possible outcomes. A possible therapy proposal based on the EOSS is shown in figure 2. 


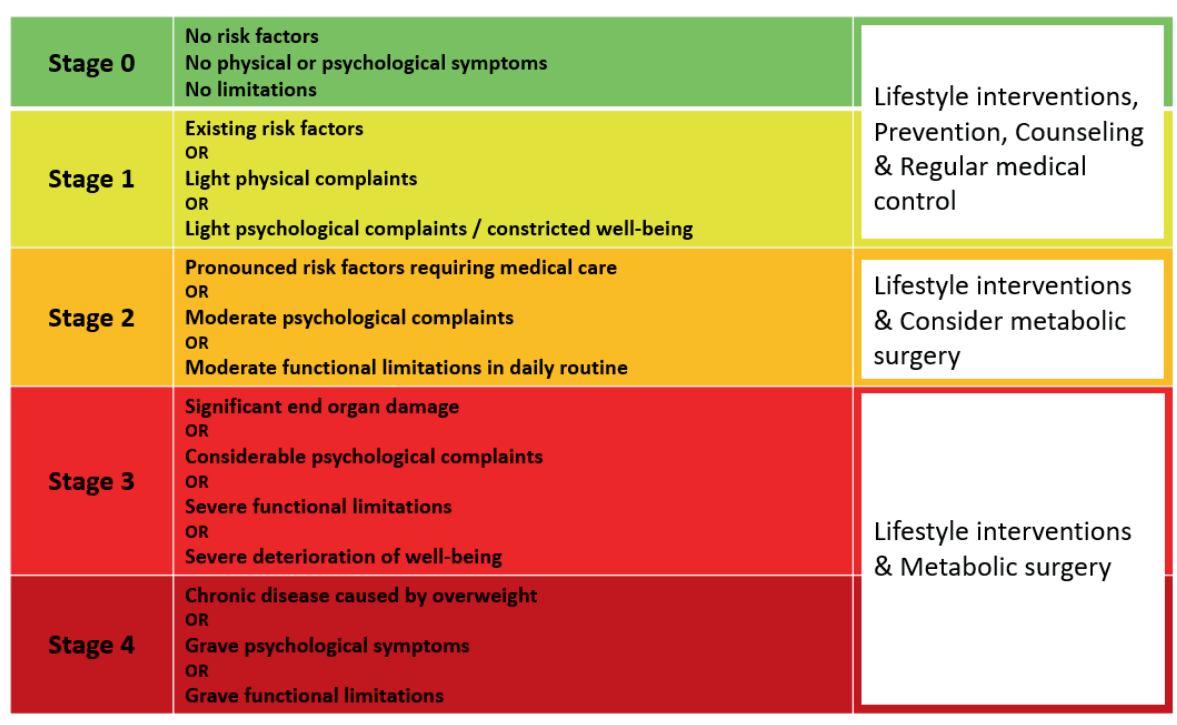

Fig. 2. Edmonton Obesity Staging System (EOSS) - evaluation of risk and therapy proposal (modified from [61]).

Ideally, medical societies should collectively define an EOSS grade for each obesity-related comorbidity, with future studies aimed toward evaluating the effectiveness of a variety of therapies and the risk-benefit ratio for each stage and disease. Based on these findings, clear indications for conservative therapy or metabolic surgery could then be defined.

\section{Indications for Metabolic Surgery for Type 2 Diabetes Mellitus}

T2DM has become a major problem worldwide and poses one of the largest challenges for health care. Despite its already high and ever increasing incidence and the high costs related to it, T2DM remains poorly understood, and there has been no improvement in the number of patients suffering from T2DM worldwide. The different conservative treatment options available at the moment range from lifestyle modifications such as increased physical activity or dietary education to pharmacotherapies including a combination of several different drugs. All of these methods, even in combination, only show a poor reduction in T2DM-related complications, including micro- and macrovascular complications as well as mortality [21]. In comparison, metabolic surgery is a very effective treatment option for T2DM as it reduces the development of micro- and macrovascular complications and mortality $[6-8,10$, 22-24]. Several meta-analyses show the superiority of metabolic surgery for T2DM remission and glycemic control in comparison to medical therapies. Diabetic remission has been achieved for obese patients undergoing biliopancreatic diversion (BPD), SG, adjustable gastric band, and RYGB [3, 4]. In an earlier meta-analysis of three observational controlled studies (OCS) and five RCTs, it has been shown that metabolic surgery achieves superior glycemic control and T2DM remission even in none-severely obese patients with a BMI $<35 \mathrm{~kg} / \mathrm{m}^{2}$ (fig. 3) [3].

In 2016, Rubino et al. [4] showed in a meta-analysis of 15 studies based on the 2nd Diabetes Surgery Summit (DSS-II) that glyce-

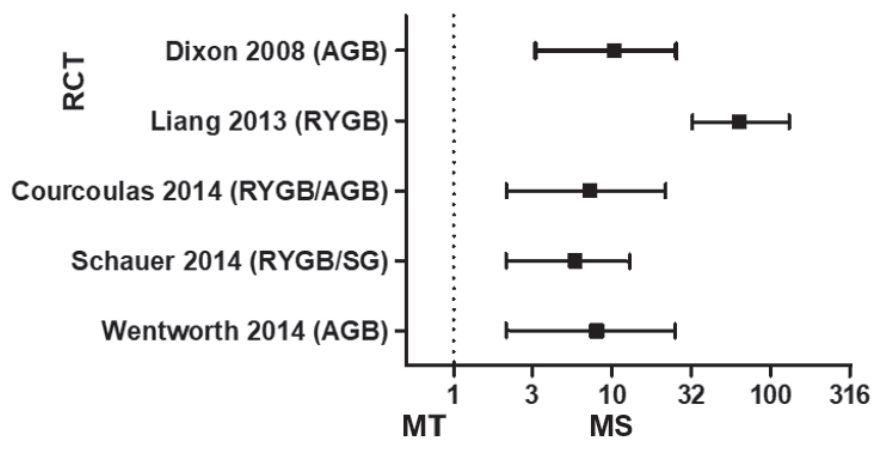

Fig. 3. RCTs demonstrating the effect of metabolic surgery on type 2 diabetes remission (modified from [3]). RCT $=$ Randomized controlled trial; AGB = adjustable gastric band; RYGB = Roux-en-Y gastric bypass; SG = sleeve gastrectomy; $\mathrm{MT}=$ medical therapy; $\mathrm{MS}=$ metabolic surgery.

mic control was more favorable following surgery than with medical therapy and lifestyle change independent of BMI. 7 of 16 studies investigated hyperglycemia in patients with a BMI $\leq 35 \mathrm{~kg} / \mathrm{m}^{2}$; 8 studies focused on individuals with a BMI $>35 \mathrm{~kg} / \mathrm{m}^{2}$. This metaanalysis clearly shows that improvement in glycemic control is independent of how overweight a patient is, and that surgery is beneficial for those who fall into either of those two groups. In the same meta-analysis, Rubino et al. [4] were able to prove the superiority of metabolic surgery regarding the diminution of glycated hemoglobin.

Regarding the time point of surgery, a recent study investigating the benefits of surgery at different stages of diabetes progression showed that patients with pre-diabetes who had surgery received the clearest benefit regarding the development of microvascular complications [18]. These findings led to the newest German S3 guideline from the German Association of General and Visceral Surgery, which were also approved by the German Diabetes Association, for 'surgery of obesity and metabolic diseases' which grant a much bigger role for metabolic surgery in the treatment of T2DM in obese patients [14]. Metabolic surgery should be recommended to patients with a BMI $\geq 40 \mathrm{~kg} / \mathrm{m}^{2}$ and coexisting T2DM, irrespec- 
tive of glycemic control or the complexity of their antidiabetic medication. The newest meta-analyses show that these patients even benefit from an improvement of microvascular complications such as existing neuropathy or nephropathy [7-10]. The new S3 guidelines specified that metabolic surgery should be recommended to patients with a BMI between 35 and $40 \mathrm{~kg} / \mathrm{m}^{2}$ who do not achieve the treatment goal with medical therapy. For a BMI $\geq$ $30 \mathrm{~kg} / \mathrm{m}^{2}$ and $<35 \mathrm{~kg} / \mathrm{m}^{2}$ and coexisting T2DM, metabolic surgery should be considered if medical therapy does not achieve the target range or does not reduce disease progression. However, metabolic surgery is still recommended only in the context of scientific studies for a BMI $<30 \mathrm{~kg} / \mathrm{m}^{2}$ and coexisting T2DM.

\section{Microvascular Complications}

Research has demonstrated that approximately $40 \%$ of patients with T2DM develop long-term microvascular complications despite consequent therapy and good glycemic control. Typical microvascular complications are kidney, retinal, and nerve damage. However, the underlying causes of these microvascular complications remain poorly understood and may even differ between the affected organs. Diabetic nephropathy is the most frequent cause of kidney disease in patients needing dialysis and has a prevalence of $40 \%$ in patients with type 1 and 2 diabetes $[25,26]$. It is thus indispensable to treat or prevent microvascular complications effectively. Ruospo et al. [21] showed in their Cochrane meta-analysis that tight glycemic control with a target of glycated hemoglobin between 6.5 and 7.5\% does not improve the risk of kidney failure, major cardiovascular events, or death compared to non-tight glycemic control.

Therefore, tight glycemic control has no relevant effect on the development of diabetic complications. In contrast to these findings, we were able to show the benefits of metabolic surgery (RYGB) in patients with insulin-dependent T2DM and a BMI between 25 and $35 \mathrm{~kg} / \mathrm{m}^{2}$ who do not achieve the glycemic target with medical therapy. RYGB reduced serum creatinine levels in the first 12 months, which remained stable at normal levels for 24 months, and resulted in an increasing glomerular filtration rate and amelioration of the microalbuminuria within 24 months, indicating a profound recovery of kidney function [7].

A meta-analysis evaluating 10 studies (3 RCTs and 7 OCS) found that metabolic surgery can prevent the development of microvascular complications better than conservative treatment and is even able to improve already existing diabetic nephropathy (fig. 4) [8]. These results demonstrate that metabolic surgery, e.g. RYGB, is an effective treatment option for overweight patients with diabetic nephropathy. Another important and common microvascular complication of T2DM is diabetic neuropathy, which has a lifetime incidence of about $45 \%$ in T2DM patients and of $54-59 \%$ in those with type 1 diabetes (T1DM) [27]. One of our own studies showed that diabetic neuropathy improves and can even disappear after metabolic surgery, independent of changes in glycated hemoglobin [9]. Importantly, improvement in diabetic neuropathy was observed within the first week after surgery and had no correlation with improvement in glycemic control or

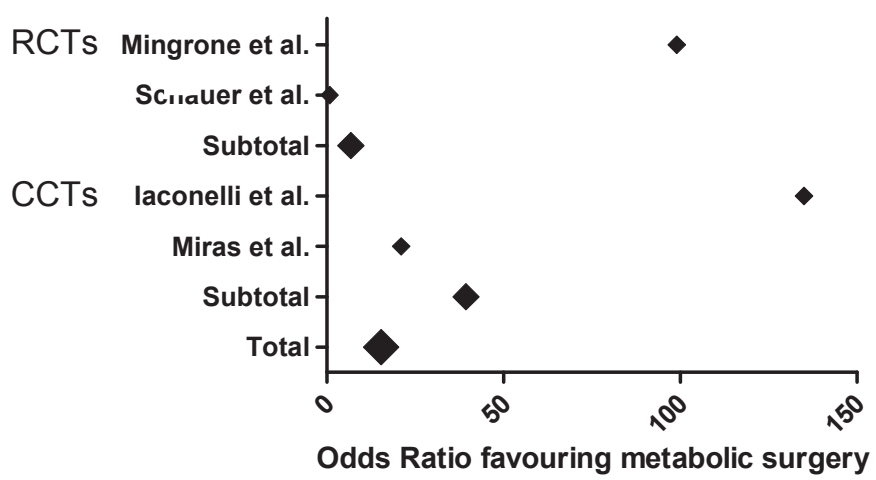

Fig. 4. Remission of nephropathy in patients with type 2 diabetes mellitus after metabolic surgery and medical therapy (modified from [8]). RCT = Randomized controlled trial; CCT = clinical controlled trial.

weight loss. However, the reduction in oxidative stress after metabolic surgery showed a strong correlation with improved diabetic neuropathy [10]. Lastly, there is also evidence that metabolic surgery reduces the incidence of diabetic retinopathy, although its effects on existing diabetic retinopathy remain elusive [8].

\section{Cardiovascular Events}

As cardiovascular disease and T2DM are closely linked, one might think that treating one would have an impact on the other. The Look AHEAD trial, however, shows that improved glycemic control combined with weight loss and improved physical fitness (or more intensive lifestyle change) does not result in a reduction of cardiovascular events [28]. Noteworthy, participants of the Look AHEAD trial who lost more than $10 \%$ of their initial body weight had significant cardiovascular benefits, suggesting an association between the magnitude of weight loss and the incidence of cardiovascular disease in people with T2DM [29]. An RCT by the Action to Control Cardiovascular Risk in Diabetes Study Group (ACCORD), which investigated over 10,250 patients, found that intensive T2DM therapy for 3.5 years actually increases mortality rates and does not reduce major cardiovascular complications such as myocardial infarction or stroke [30].

In an RCT of 60 patients, Mingrone et al. [5] assessed the cardiovascular risk in patients treated conservatively or surgically (with gastric bypass and BPD). They found that the risk diminished in all three groups, but patients who underwent surgery only had half the cardiovascular risk after 5 years than those who had received medical treatment. In their 5-year follow-up period, Schauer et al. [6] noted a reduction in the need for cardiovascular medication after metabolic surgery. Sjöström et al. [23] demonstrated in the Swedish obese subjects (SOS study) that after metabolic surgery (whether gastric bypass, banding, or vertical banded gastroplasty), the number of cardiovascular deaths and the incidence of cardiovascular events was significantly diminished overall. Halperin et al. [31] conducted an RCT that yielded comparable results: non-fatal and fatal chronic heart disease, as calculated by the UKPDS (United Kingdom Prospective Diabetes Study) Risk Engine, decreased significantly after RYGB. 
Fig. 5. Overview over proposed Edmonton Obesity Staging System of type 2 diabetes mellitus, non-alcoholic fatty liver disease, and non-alcoholic steatohepatitis (NASH). HCC $=$ Hepatocellular carcinoma.

\begin{tabular}{|c|l|l|}
\hline EOSS & T2DM & NAFLD/NASH \\
\hline 0 & No glucose intolerance & No steatosis \\
\hline 1 & Impaired glucose tolerance & Steatosis without inflammatory reaction \\
\hline 2 & Glucose intolerance medically treated & Beginning NASH without fibrosis \\
\hline 3 & Micro- or macrovascular complications & NASH with fibrosis \\
\hline 4 & Organ failure & Liver cirrhosis or HCC \\
\hline
\end{tabular}

\section{Proposed Use of EOSS Grading for T2DM}

When applying the EOSS stages to T2DM, stage 0 would correspond to normal glucose metabolism while stage 1 would correspond to an impaired glucose tolerance. Patients requiring medical treatment will be staged EOSS 2 if they do not have any micro- or macrovascular complications. Subsequent micro- or macrovascular complications such as neuropathy, nephropathy, retinopathy, acute coronary disease, or previous myocardial infarction would correspond to EOSS 3. In cases of organ failure such as renal insufficiency or decreased ventricular function, an EOSS of 4 would apply. Based on these findings, metabolic surgery would be indicated from EOSS 2 on, and may even be considered for patients with EOSS 1 plus additional risk features for disease progression. Figure 5 shows an overview of the proposed EOSS staging.

\section{Indications for Metabolic Surgery for NAFLD/NASH}

Non-alcoholic fatty liver disease (NAFLD) is a rapidly increasing chronic liver disease. It is most prevalent in individuals over the age of 60 years in industrialized countries. T2DM and NAFLD are highly connected through obesity. Together with the obesity pandemic, NAFLD and T2DM have become more frequent worldwide [32-34]. NAFLD can progress, as determined by the histological features of lobular inflammation, hepatocyte ballooning, and necrosis to NASH, which then can progress to fibrosis and cirrhosis. The increasing indication for liver transplantation due to NASH cirrhosis or the high risk that NASH, even in its fibrotic and not yet cirrhotic state, can develop into a hepatocellular carcinoma (HCC) shows that NAFLD and NASH should be treated both early and efficiently [35-37]. Of note, T2DM itself is a risk factor for the development of HCC, independent of the underlying liver disease [38].

So far, the only effective conservative therapy for NAFLD and NASH is weight reduction [39]. Because conservative weight loss methods offer minimal short-term benefit and almost always end in patients regaining weight, it is crucial to find a permanent means of weight loss for patients who are at risk for or already suffering from NAFLD or NASH. In Germany, there is no drug approved for the therapy of NAFLD/NASH, although several drugs are currently under investigation $[40,41]$. Novel pharmacotherapies are being researched intensively although their effectiveness thus far remains doubtful. Birkenfeld et al. [42] have defined different targets in the development of NASH, the most important of which include insulin resistance, lipotoxicity, oxidative stress, and altered regulation of immunity. One way of reducing insulin resistance is by administer- ing insulin sensitizers like glitazones. Glitazones, agonists of the peroxisome proliferator-activated receptor (PPAR $\gamma$ ) which modulate glucose and lipid homeostasis as well as inflammation, can be used as a treatment for T2DM [43]. Some beneficial effects of glitazone administration are a reduction in insulin resistance and $\mathrm{HbA1c}$ as well as improvement of liver damage caused by NASH. The latter effect is caused, among other reasons, by the diminution of hepatic glucose production and, subsequently, that of steatosis. Unfortunately, the practical use of glitazones is limited due to their frequent and often severe side effects such as congestive heart failure, bone fractures, and bladder cancer. However, metformin, despite also improving insulin sensitivity, does not have a significant effect on NASH. Antioxidative agents such as vitamin E only have modest effects on NAFLD and no effect on NASH.

In contrast, metabolic surgery is a very effective treatment for NAFLD in general but in particular for NASH. A recent study by Lassailly et al. [44] showed the beneficial and significant changes in steatosis and NAFLD activity score (NAS) after metabolic surgery (fig. 6): steatosis decreased from 60 to $10 \%$ after surgical treatment, and the NAS diminished from 5 to 1 , essentially normalizing the liver histology in patients with NASH. Interestingly, the authors even showed that metabolic surgery can reduce liver fibrosis. Another meta-analysis also demonstrated this effect: each of the 16 studies included demonstrated a diminution of steatosis after metabolic surgery [45].

Metabolic surgery does not only improve NASH but also has a profound effect on disease-related mortality. McCarty et al. [46] analyzed data on 45,462 patients with liver cirrhosis who were included in the HICUP database for the years 2004-2012 and found a large difference in mortality depending on whether a patient had metabolic surgery or not (5\% with and $69 \%$ without metabolic surgery). Although such studies are often skewed due to the nature of administrative databases, this result still shows that metabolic surgery seems to have a profound impact on the progression of liver disease.

Obese patients also have an overall higher rate of cancer. One meta-analysis showed that metabolic surgery was able to decrease the likelihood of cancer by reducing weight effectively [47]. The incidence of liver cancer in particular was reduced by metabolic surgery, as NAFLD and NASH, both common among obese patients, may progress to HCC [48]. Applying the EOSS to NAFLD or NASH, stage 1 corresponds to steatosis without inflammatory reaction. EOSS 2 would then be applied to patients with histological features of NASH without fibrosis, and EOSS 3 to cases of NASH with fibrosis. Liver cirrhosis or HCC would fulfill criteria for EOSS 4 (fig. 5). 
Fig. 6. Changes in non-alcoholic steatohepatitis inflammatory activity grade after metabolic surgery (modified from [44]).

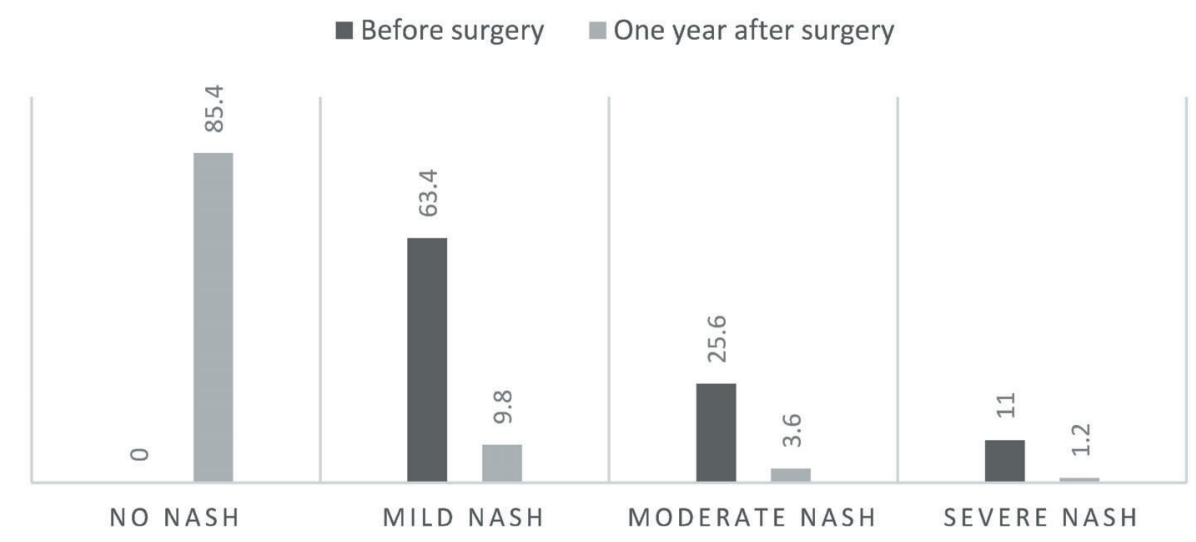

\section{Metabolic Surgery for Other Potential Indications}

Dyslipidemia, frequently characterized by high circulating triglycerides and low high-density lipoprotein cholesterol, is another common comorbidity of obesity. Bays et al. [49], when analyzing the Study to Help Improve Early evaluation and management of risk factors Leading to Diabetes (SHIELD) and National Health and Human Nutrition Examination Surveys (NHANES) databases, found that an increase in BMI is associated with an increased prevalence of dyslipidemia. Dimitri et al. [50] showed a decrease in the prevalence of dyslipidemia after metabolic surgery. This effect was significant after the first year and remained stable throughout the 5-year follow-up. In an earlier meta-analysis, we also found that metabolic surgery leads to a reduction of dyslipidemia independent of the surgical techniques used [3].

Obstructive sleep apnea syndrome (OSAS) is often associated with obesity. Approximately $42-48 \%$ of obese men and $8-38 \%$ of obese women suffer from OSAS [51]. OSAS increases the risk of stroke, lowers quality of life, and is also common in patients with heart failure [52,53]. Treatment of OSAS includes continued positive airway pressure, oral appliance, and upper airway surgery [5456]. In a retrospective study with a 5-year follow up, Dimitri et al. [50] showed that the prevalence of sleep apnea decreases significantly after metabolic surgery. A systematic review comparing 19 surgical and 20 non-surgical studies similarly proved that metabolic surgery has more impact on the apnea-hypopnea index than non-surgical weight loss does [57]. Comparing different surgical methods, Sarkhosh et al. [58] found that every metabolic surgery procedure was highly effective in improving OSAS although 25\% of patients reported no improvement at all.

Obesity-related glomerulopathy (ORG) is defined by glomerulomegaly with or without focal and segmental glomerulosclerosis lesions and is caused by obesity independent of T2DM [59]. Obesity also increases the risk of hypertension, which in turn increases the risk of chronic kidney disease by vasoconstriction as well as electrolyte and water retention [60]. Furthermore, it increases intrarenal inflammation. At present, no definitive treatment has been defined for ORG, although aggressive weight loss through metabolic surgery seems promising.
Further RCTs are needed to investigate which patients' criteria and which stage of dyslipidemia, OSAS, and ORG predict the best outcome after metabolic surgery. Additionally, more data are needed before these three diseases can be included in the EOSS.

\section{Conclusion}

Metabolic surgery is an effective treatment for various metabolic diseases including T2DM, NAFLD/NASH, dyslipidemia, and others. Importantly, there is overwhelming evidence that the beneficial effects of metabolic surgery are not dependent on preoperative BMI and that it is similarly effective for patients with a BMI < $35 \mathrm{~kg} / \mathrm{m}^{2}$. Hence, we propose that the EOSS should be used as a staging tool for metabolically sick patients, and that therapy stratification be based on EOSS stages. Evidence consistently shows that metabolic surgery is the most effective therapy for reducing overall as well as cardiovascular-related mortality in obese patients with metabolic disease. Further, metabolic surgery does not only reduce disease incidence but also improves existing microvascular complications, is highly effective in reducing the histological features of $\mathrm{NASH}$, and reverses fibrosis.

Based on these findings, metabolic surgery should be a mainstay in the treatment of metabolic disease. Healthcare providers treating patients with such diseases should define and agree on how EOSS stages should be utilized best and at which stages metabolic surgery should be recommended.

\section{Author Contributions}

Concept and design: ATB, BPM and MB. Data acquisition (literature search): ATB, ACS and KMS. Drafting of the manuscript: ATB, ACS. Critical revisions: BPM, MB, ATB and KMS.

\section{Disclosure Statement}

This research did not receive any specific funding from any funding agencies in the public, commercial or non-profit sectors.

The authors have no conflicts of interest to declare. 


\section{References}

1 Pories WJ, et al: Who would have thought it? An operation proves to be the most effective therapy for adultonset diabetes mellitus. Ann Surg 1995;222:339-350; discussion 350-352

2 Sjöström L, et al: Association of bariatric surgery with long-term remission of type 2 diabetes and with microvascular and macrovascular complications. JAMA 2014; 311:2297-2304.

3 Müller-Stich BP, et al: Surgical versus medical treatment of type 2 diabetes mellitus in nonseverely obese patients: a systematic review and meta-analysis. Ann Surg 2015; 261:421-429.

4 Rubino F, et al: Metabolic surgery in the treatment algorithm for type 2 diabetes: a joint statement by international diabetes organizations. Diabetes Care 2016;39: 861-877.

5 Mingrone G, et al: Bariatric-metabolic surgery versus conventional medical treatment in obese patients with type 2 diabetes: 5 year follow-up of an open-label, single-centre, randomised controlled trial. Lancet 2015;386:964-973.

6 Schauer PR, et al: Bariatric surgery versus intensive medical therapy for diabetes - 5-year outcomes. N Engl J Med 2017;376:641-651.

7 Billeter AT, et al: Renal function in type 2 diabetes following gastric bypass. Dtsch Arztebl Int 2016;113:827833.

8 Billeter AT, et al: Meta-analysis of metabolic surgery versus medical treatment for microvascular complications in patients with type 2 diabetes mellitus. Br J Surg 2018; 105:168-181.

9 Müller-Stich BP, et al: Gastric bypass leads to improvement of diabetic neuropathy independent of glucose normalization - results of a prospective cohort study (DiaSurg 1 study). Ann Surg 2013;258:760-765; discussion 765-766.

10 Müller-Stich BP, et al: Nitrosative stress but not glycemic parameters correlate with improved neuropathy in nonseverely obese diabetic patients after Roux-Y gastric bypass. Surg Obes Relat Dis 2015;11:847-854.

11 Blüher M: The distinction of metabolically 'healthy' from 'unhealthy' obese individuals. Curr Opin Lipidol 2010; 21:38-43.

12 Blüher M: Are there still healthy obese patients? Curr Opin Endocrinol Diabetes Obes 2012;19:341-346.

13 Stefan N, et al: Metabolically healthy obesity: epidemiology, mechanisms, and clinical implications. Lancet Diabetes Endocrinol 2013;1:152-162.

14 Deutsche Gesellschaft für Allgemein- und Viszeralchirurgie e.V. (DGAV), Deutsche Adipositas-Gesellschaft (DAG); Deutsche Diabetes Gesellschaft (DDG), Deutsche Gesellschaft für Ernährungsmedizin e.V. (DGEM), Deutsche Gesellschaft für Endoskopie und Bildgebende Verfahren (DGE-BV), Deutsche Gesellschaft für Psychosomatische Medizin und Ärztliche Psychotherapie e.V. (DGPM), Deutsche Gesellschaft der Plastischen, Rekonstruktiven und Ästhetischen Chirurgen (DGPRÄC), Deutsches Kollegium für Psychosomatische Medizin (DKPM), Verband der Diabetesberatungs- und Schulungsberufe in Deutschland e.V. (VDBD); BerufsVerband Oecotrophologie e.V. (VDOE); Adipositaschirurgie-SelbsthilfeDeutschland e.V.: S3-Leitlinie: Chirurgie der Adipositas und metabolischer Erkrankungen. Version 2.3 (Februar 2018), AWMF-Register Nr. 088-001. 2018. https://www. awmf.org/uploads/tx_szleitlinien/088-0011_S3_ChirurgieAdipositas-metabolische-Erkrankugen_2018-02.pdf.

15 Caleyachetty R, et al: Metabolically healthy obese and incident cardiovascular disease events among 3.5 million men and women. J Am Coll Cardiol 2017;70:1429-1437.

16 Sharma AM, Kushner RF: A proposed clinical staging system for obesity. Int J Obes (Lond) 2009;33:289-295.

17 Padwal RS, et al: Using the Edmonton obesity staging system to predict mortality in a population-representative cohort of people with overweight and obesity. CMAJ 2011;183:E1059-1066.
Carlsson LMS, et al: Long-term incidence of microvascular disease after bariatric surgery or usual care in patients with obesity, stratified by baseline glycaemic status: a post-hoc analysis of participants from the Swedish Obese Subjects study. Lancet Diabetes Endocrinol 2017;5:271279.

19 Jimenez A, et al: Long-term effects of sleeve gastrectomy and Roux-en-Y gastric bypass surgery on type 2 diabetes mellitus in morbidly obese subjects. Ann Surg 2012;256: 1023-1029.

20 Ahlqvist E, et al: Novel subgroups of adult-onset diabetes and their association with outcomes: a data-driven cluster analysis of six variables. Lancet Diabetes Endocrinol 2018;6:361-369.

21 Ruospo M, et al: Glucose targets for preventing diabetic kidney disease and its progression. Cochrane Database Syst Rev 2017;6:CD010137.

22 Christou NV, et al: Surgery decreases long-term mortality, morbidity, and health care use in morbidly obese patients. Ann Surg 2004;240:416-423; discussion 423-424.

23 Sjöström L, et al: Bariatric surgery and long-term cardiovascular events. JAMA 2012;307:56-65.

24 Adams TD, et al: Long-term mortality after gastric bypass surgery. N Engl J Med 2007;357:753-761.

25 Gross JL, et al: Diabetic nephropathy: diagnosis, prevention, and treatment. Diabetes Care 2005;28:164-176.

26 Molitch ME, et al: Diabetic nephropathy. Diabetes Care 2003;26(suppl 1):S94-98.

27 Zilliox L, Russell JW: Treatment of diabetic sensory polyneuropathy. Curr Treat Options Neurol 2011;13:143159.

28 Look AHEAD Research Group; Wing RR, et al: Cardiovascular effects of intensive lifestyle intervention in type 2 diabetes. N Engl J Med 2013;369:145-154.

29 Gregg EW, et al: Association of the magnitude of weight loss and changes in physical fitness with long-term cardiovascular disease outcomes in overweight or obese people with type 2 diabetes: a post-hoc analysis of the Look AHEAD randomised clinical trial. Lancet Diabetes Endocrinol 2016;4:913-921.

30 Action to Control Cardiovascular Risk in Diabetes Study Group; Gerstein HC, et al: Effects of intensive glucose lowering in type 2 diabetes. N Engl J Med 2008;358: 2545-2559.

31 Halperin F, et al: Roux-en-Y gastric bypass surgery or lifestyle with intensive medical management in patients with type 2 diabetes: feasibility and 1-year results of a randomized clinical trial. JAMA Surg 2014;149:716-726.

32 Loomba R, Sanyal AJ: The global NAFLD epidemic. Nat Rev Gastroenterol Hepatol 2013;10:686-690.

33 Wong RJ, Ahmed A: Obesity and non-alcoholic fatty liver disease: disparate associations among Asian populations. World J Hepatol 2014;6:263-273.

34 Chalasani N, et al: The diagnosis and management of non-alcoholic fatty liver disease: practice Guideline by the American Association for the Study of Liver Diseases, American College of Gastroenterology, and the American Gastroenterological Association. Hepatology 2012;55:2005-2023.

35 Agopian VG, et al: Liver transplantation for nonalcoholic steatohepatitis: the new epidemic. Ann Surg 2012; 256:624-633.

36 Charlton MR, et al: Frequency and outcomes of liver transplantation for nonalcoholic steatohepatitis in the United States. Gastroenterology 2011;141:1249-1253.

37 Wong RJ, et al: Nonalcoholic steatohepatitis is the sec ond leading etiology of liver disease among adults awaiting liver transplantation in the United States. Gastroenterology 2015;148:547-555.

38 Wang C, et al: Increased risk of hepatocellular carcinoma in patients with diabetes mellitus: a systematic review and meta-analysis of cohort studies. Int J Cancer 2012; 130:1639-1648.
9 Vilar-Gomez E, et al: Weight loss through lifestyle modification significantly reduces features of nonalcoholic steatohepatitis. Gastroenterology 2015;149:367-378.e5; quiz e14-15.

40 Musso G, Cassader M, Gambino R: Non-alcoholic steatohepatitis: emerging molecular targets and therapeutic strategies. Nat Rev Drug Discov 2016;15:249-274.

41 Ratziu V, Goodman Z, Sanyal A: Current efforts and trends in the treatment of NASH. J Hepatol 2015;62 (suppl):S65-75.

42 Birkenfeld AL, Shulman GI: Nonalcoholic fatty liver disease, hepatic insulin resistance, and type 2 diabetes. Hepatology 2014;59:713-723.

43 Cariou B, Charbonnel B, Staels B: Thiazolidinediones and PPARgamma agonists: time for a reassessment. Trends Endocrinol Metab 2012;23:205-215.

44 Lassailly G, et al: Bariatric surgery reduces features of nonalcoholic steatohepatitis in morbidly obese patients. Gastroenterology 2015;149:379-388; quiz e15-16.

45 Bower G, et al: Bariatric surgery and non-alcoholic fatty liver disease: a systematic review of liver biochemistry and histology. Obes Surg 2015;25:2280-2289.

46 McCarty TR, et al: Impact of bariatric surgery on outcomes of patients with nonalcoholic fatty liver disease: a nationwide inpatient sample analysis, 2004-2012. Surg Obes Relat Dis 2018;14:74-80.

47 Casagrande DS, et al: Incidence of cancer following bariatric surgery: systematic review and meta-analysis. Obes Surg 2014;24:1499-1509.

48 Yang B, et al: Bariatric surgery and liver cancer in a consortium of academic medical centers. Obes Surg 2016;26: 696-700.

49 Bays HE, Chapman RH, Grandy S: The relationship of body mass index to diabetes mellitus, hypertension and dyslipidaemia: comparison of data from two national surveys. Int J Clin Pract 2007;61:737-747.

50 Dimitri Miras A, et al: Obesity surgery makes patients healthier and more functional: real world results from the United Kingdom National Bariatric Surgery Registry. Surg Obes Relat Dis 2018;14:1033-1040.

51 Young T, et al: The occurrence of sleep-disordered breathing among middle-aged adults. N Engl J Med 1993;328:1230-1235.

52 Mohsenin V: Is sleep apnea a risk factor for stroke? A critical analysis. Minerva Med 2004;95:291-305.

53 Sharma B, McSharry D, Malhotra A: Sleep disordered breathing in patients with heart failure: pathophysiology and management. Curr Treat Options Cardiovasc Med 2011;13:506-516.

54 Wright J, White J: Continuous positive airways pressure for obstructive sleep apnoea. Cochrane Database Syst Rev 2000;(2):CD001106.

55 Lim J, et al: Oral appliances for obstructive sleep apnoea. Cochrane Database Syst Rev 2003;(4):CD004435.

56 Sundaram S, et al: Surgery for obstructive sleep apnoea. Cochrane Database Syst Rev 2005;(4):CD001004.

57 Ashrafian $\mathrm{H}$, et al: Bariatric surgery or non-surgical weight loss for obstructive sleep apnoea? A systematic review and comparison of meta-analyses. Obes Surg 2015;25:1239-1250.

58 Sarkhosh K, et al: The impact of bariatric surgery on obstructive sleep apnea: a systematic review. Obes Surg 2013;23:414-423.

59 Xu T, Sheng Z, Yao L: Obesity-related glomerulopathy: pathogenesis, pathologic, clinical characteristics and treatment. Front Med 2017;11:340-348.

60 Snyder S, Turner GA, Turner A: Obesity-related kidney disease. Prim Care 2014;41:875-893.

61 IFB Adipositas Erkrankungen, Universitätsmedizin Leipzig: Adipositas Management. Ein Leitfaden für Behandler. Leipzig, 2015. www.ifb-adipositas.de/sites/ default/files/5a-adipositas-management-manual-fuer-behandler_0.pdf. 